Article

\title{
Surface Enhanced Raman Scattering (SERS) Studies of Gold and Silver Nanoparticles Prepared by Laser Ablation
}

\author{
Gloria M. Herrera, Amira C. Padilla and Samuel P. Hernandez-Rivera * \\ ALERT-DHS Center of Excellence/Center for Chemical Sensors Development, \\ University of Puerto Rico-Mayagüez, P.O. Box 9000, Mayaguez, PR 00681-9000, USA; \\ E-Mails: gloriam.herrera@upr.edu (G.M.H.); amirac.padilla@upr.edu (A.C.P.) \\ * Author to whom correspondence should be addressed; E-Mail: samuel.hernandez3@upr.edu; \\ Tel.: +1-787-265-5458; Fax: +1-787-265-5404.
}

Received: 15 December 2012; in revised form: 1 February 2013 / Accepted: 7 February 2013 / Published: 1 March 2013

\begin{abstract}
Gold and silver nanoparticles (NPs) were prepared in water, acetonitrile and isopropanol by laser ablation methodologies. The average characteristic (longer) size of the NPs obtained ranged from 3 to $70 \mathrm{~nm}$. 4-Aminobenzebethiol (4-ABT) was chosen as the surface enhanced Raman scattering (SERS) probe molecule to determine the optimum irradiation time and the $\mathrm{pH}$ of aqueous synthesis of the laser ablation-based synthesis of metallic NPs. The synthesized NPs were used to evaluate their capacity as substrates for developing more analytical applications based on SERS measurements. A highly energetic material, TNT, was used as the target compound in the SERS experiments. The Raman spectra were measured with a Raman microspectrometer. The results demonstrate that gold and silver NP substrates fabricated by the methods developed show promising results for SERS-based studies and could lead to the development of micro sensors.
\end{abstract}

Keywords: laser ablation; Au and Ag NP; Raman spectroscopy; SERS; explosives

\section{Introduction}

The many uses of nanotechnology include very important applications in areas, such as medicine [1-5], catalysis [6-14], industrial applications [15-17] and scientific investigations. The size, shape and physicochemical properties are very important in future applications and are the main theme in studies currently conducted. There are several ways to synthesize metal nanoparticles (NPs). Their 
synthesis can be classified as either chemical or physical methods. Some chemical methods include the chemical reduction of metal salts, the alcohol reduction process, the polyol process, microemulsions, the thermal decomposition of metal salts and electrochemical synthesis [18,19]. Physical methods include pulsed laser ablation, the exploding wire technique, plasma, chemical vapor deposition, microwave irradiation, supercritical fluids, sono-chemical reduction and gamma radiation [20]. Laser ablation is a very clean physical method for the preparation of metallic nanoparticles (NPs). During the last year, the laser ablation of metals has increased in popularity, due to the fast and simple nature of the procedure. In addition, the important advantage of this method when compared to chemical synthesis ("wet chemistry syntheses") is the preparation of high surface purity NPs in the chosen solvent. There are no counter ions and no residuals of the reducing agents remaining on the surfaces of the NPs [21-23]. To take advantage of these features, we have prepared gold (Au) and silver (Ag) NPs by laser ablation. Colloidal suspensions of prepared NPs were deposited on gold-coated slides to immobilize them and to test them for potential use as substrates for the detection of explosives using surface enhanced Raman scattering (SERS).

The use of spectroscopic techniques with intensities augmented by nanostructured metal surfaces has attracted great interest in recent years. The SERS effect discovered in the seventies is largely attributed to the interaction of light with matter. Specifically, SERS is related to the inelastic scattering (or Raman scattering) of certain molecules in the presence of specially prepared roughened or discontinuous metallic nanostructures. There are two mechanisms that explain the increase in the Raman signal. The first is explained through an electromagnetic interaction model (EM) and the second through a chemical interaction model or charge transfer (CT) [24,25]. Both mechanisms are thought to contribute to the signal intensity enhancement observed, although the extent of the contribution of each source of enhancement depends on the system under study. The enhancement mechanisms of the Raman signal lead to a technique with a sensitivity and selectivity that make Raman scattering a highly promising technique for further developing analytical applications [26-31]. These applications are closely related to the properties and the surface morphology of the metallic NP used. A large part of the contribution to the SERS effect is due to the increase in the inelastically scattered Raman signal intensity by the nanostructured metal systems present and the particular properties of the particles to induce greater morphological coupling with the incident radiation, resulting in intense spectroscopic signals. The molecule-surface relative orientation allows the emergence of new selection rules, resulting in the intensification of the Raman spectrum bands corresponding to the molecular vibrations of the molecular polarizability components perpendicular to the surface. The Au and Ag NPs prepared by laser ablation were deposited on various substrates and subsequently evaluated as SERS substrates, with the objective of detecting explosives, such as TNT.

\section{Experimental Section}

\subsection{Laser Ablation Synthesis}

Au or Ag metal foils (99.99\%, Sigma-Aldrich, Milwaukee, WI, USA) were placed in a vial containing $10 \mathrm{~mL}$ of deionized water as ablation and heat tempering media. Laser pulses at $1064 \mathrm{~nm}$, obtained using a Quanta-Ray Pro Series pulsed Nd:YAG laser from Spectra-Physics/Newport 
Corporation (Mountain View, CA, USA), were used to ablate the metallic foils. The laser was operated in single-shot mode $(5 \mathrm{~ns}, 10 \mathrm{~Hz}$ ). The target was irradiated using a focusing lens with a focal length of $86.4 \mathrm{~cm}$. The laser power used was $0.980 \mathrm{~mW}$, and the energy was $106 \mathrm{~mJ}$. The ablation process was carried out for time intervals of 5, 10, 15 and 20 min of near IR laser pulse irradiation.

\subsection{Characterization of NPs Suspensions}

A UV-Vis spectrophotometer (Agilent model 8453, Santa Clara, CA, USA) was used to acquire the electronic absorption spectra of the NPs in water. The spectra were recorded in the range of 300 to $900 \mathrm{~nm}$. Quartz cells with a $1.0 \mathrm{~cm}$ path length (72-Q-10, obtained from Starna Cells, Inc., Atascadero, CA, USA) were used for the experiments. The NPs morphology and size were obtained from high-resolution transmission electron microscopy (HR-TEM) images (Zeiss, model 922 operated at $200 \mathrm{kV}$ ). The samples for TEM analysis were prepared by depositing $5 \mu \mathrm{L}$ of the metallic NP suspensions on ultrathin carbon film/holey carbon 400 mesh copper grids (01824 from Ted Pella, Inc., Redding, CA, USA). Zeta potential and hydrodynamic radius (HR) measurements were obtained using a Zetasizer ${ }^{\mathrm{TM}}$ Nano Series (Malvern Instruments Ltd., Worcestershire, UK).

\subsection{Effect of $p H$ on the Synthesis of NPS}

Solutions of $1 \mathrm{mM} 4-\mathrm{ABT}$ were used as analytes to evaluate the $\mathrm{pH}$ effect on the synthesis by laser ablation of colloidal suspensions of $\mathrm{Au}$ and Ag NPs. The preparation method used was as described in Section 2.1. Studies were performed only at the optimum irradiation time. Dilute solutions of $\mathrm{NaOH}$ and $\mathrm{HCl}$ were used to adjust the $\mathrm{pH}$ in the aqueous media used as the solvent in the synthesis of nanoparticles. After the synthesis at various $\mathrm{pH}$ values was studied, the UV-Vis spectra of the suspensions were obtained. The $\mathrm{pH}$ values of the aqueous colloidal suspensions used for synthesis were 2.6, 4.8, 8.1 and 10.3.

\subsection{Evaluation of SERS Activity}

SERS spectra were excited with a $514.5 \mathrm{~nm}$ INNOVA 308 Argon ion laser or a $532 \mathrm{~nm}$ VERDI 6.0 solid-state diode laser (both from Coherent, Inc., Santa Clara, CA, USA) and a $785 \mathrm{~nm}$ solid-state laser (InProcess Inc., Salt Lake City, UT, USA). 4-Aminobenzebethiol (4-ABT, Sigma-Aldrich) and 1,2-bis(4-pyridyl)ethylene (BPE, Sigma-Aldrich) were used as SERS probe analytes. Renishaw Raman Microspectrometers RM1000 and RM2000 systems (Agiltron, Inc., Woburn, MA, USA) were used to acquire normal Raman (NR) and SERS spectra. The laser power at the samples was typically in the range of 10-60 mW. The data acquisition time was $20 \mathrm{~s}$ with 2 accumulations. The spectra are presented without pre-treatments or baseline corrections.

$\mathrm{Au}$ and $\mathrm{Ag}$ NPs at different $\mathrm{pH}$ values were used to evaluate the effect of the $\mathrm{pH}$ of the colloidal suspensions on the SERS activity obtained. Thus, $1.0 \mathrm{mM}$ 4-ABT solutions and TNT solutions were used to evaluate the SERS activity at different $\mathrm{pH}$ values. Aliquots of $3 \mu \mathrm{L}$ of TNT solutions at $1.0 \mathrm{mM}$ were deposited on $\mathrm{Au} \mathrm{NP} / \mathrm{Au}$ substrates. To determine the surface enhancement factor (SEF), a solution of 4-ABT at $1.0 \times 10^{-9} \mathrm{M}$ was deposited on $\mathrm{Au} \mathrm{NP} / \mathrm{Au}$ substrates (approximately 5-10 $\mu \mathrm{L}$ of Au NPs deposited on Au substrates). 


\subsection{Detection of TNT Using Au NP/Au Substrates}

The low limit of detection (LOD) of TNT using Au NPs deposited on Au-coated glass slides and used as substrates for the NPs was calculated based on the SERS data. For the spectral measurements, aqueous solutions of 2,4,6-trinitoluene (TNT) of $1.0 \times 10^{-4} \mathrm{M}, 1.0 \times 10^{-6} \mathrm{M}$ and $1.0 \times 10^{-10} \mathrm{M}$ were used. Aliquots of $10 \mu \mathrm{L}$ of Au NPs were deposited on Au-coated substrates, and Raman spectra were measured. Then, $2.5 \mu \mathrm{L}$ of TNT solutions were deposited on Au NP films. The NPs used were prepared in acetone to facilitate the evaporation of the solvent and the quicker use of the substrate.

\section{Results and Discussion}

This contribution focuses on developing fast and simple methods for the preparation of SERS-active substrates with high sensitivity. For this purpose, NPs were synthesized using laser ablation methods. Au and Ag NP suspensions were synthesized at different irradiation times (5, 10, 15 and $20 \mathrm{~min}$ ). UV-Vis absorption measurements were obtained to characterize the NPs obtained (Figure 1A,B). The typical positions of the surface plasmon maximum absorption wavelength for $\mathrm{Au}$ NPs and Ag NPs were approximately $525 \mathrm{~nm}$ (Figure 1A) and $400 \mathrm{~nm}$ (Figure 1B), respectively. These absorption maxima correspond to spherical (or nearly spherical) NPs with a characteristic average diameter between 2 and $100 \mathrm{~nm}$ [32]. These results were corroborated with TEM images and the corresponding statistical and morphological analyses.

Figure 1. UV-Vis absorption spectra of $\mathrm{Au}$ and $\mathrm{Ag}$ nanoparticles (NPs) at various irradiation times: (A) absorption spectra of Au NPs; (B) absorption spectra of Ag NPs.

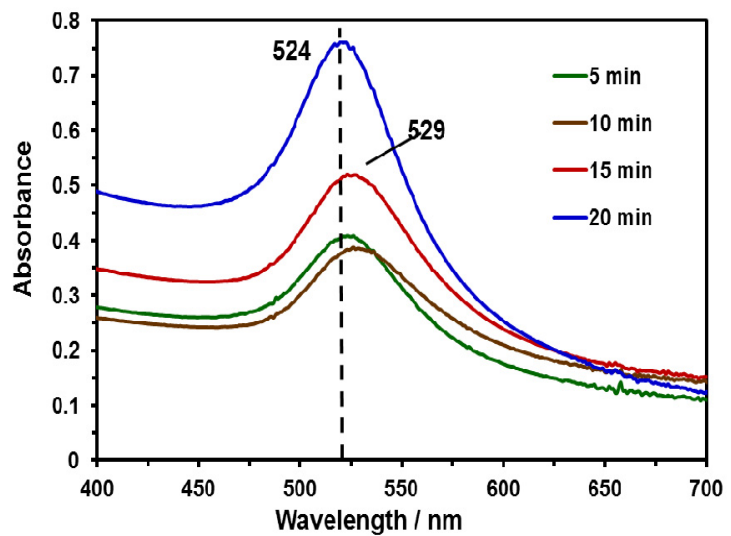

(A)

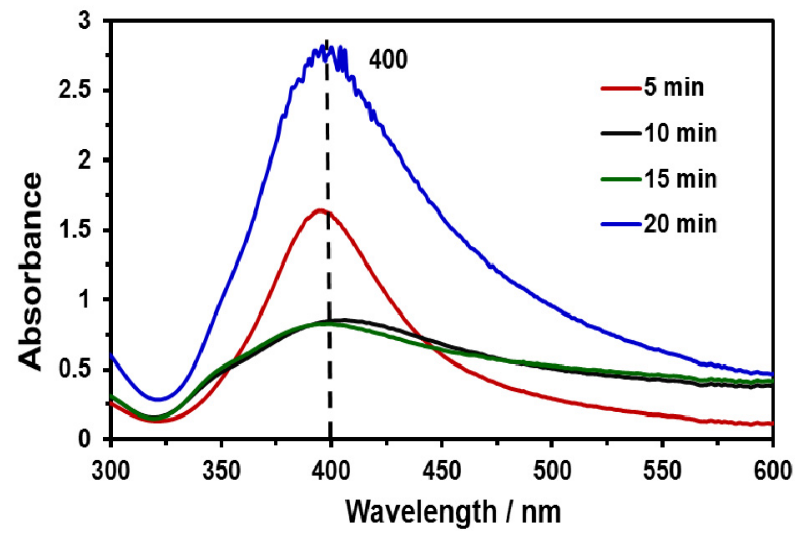

(B)

TEM images of the prepared $\mathrm{Au}$ and Ag NPs are shown in Figure 2. Colloidal suspensions of Au NPs of different sizes were obtained. Purple colloids are typical of large Au NPs of approximately $126 \pm 36 \mathrm{~nm}$ (Figure 2A). Red colloid suspensions have average sizes of $11 \pm 4 \mathrm{~nm}$ (Figure 2A). The spherical shape is predominant in the images. Similarly, Figure 2C,D show TEM images of typical colloidal suspensions of Ag NPs. Green-gray colloidal suspensions have NPs with spheroidal, large Ag NPs of approximately $132 \pm 5 \mathrm{~nm}$ and yellow Ag NPs are spherical seeds of $5 \pm 1 \mathrm{~nm}$. The average sizes of the NPs shown in these TEM images were determined using the "ImageJ" program (NIH). 
Figure 2. TEM images of $\mathrm{Au}$ and Ag NPs. Au NPs: (A) large spheres with average diameters of $126 \pm 39 \mathrm{~nm}$ are violet. (B) red colloids have average diameters of $11 \pm 4 \mathrm{~nm}$ Ag NPs: (C) yellow Ag NPs suspensions have average sizes of $132 \pm 5 \mathrm{~nm}$; (D) silver seed-like NP with an average long axis of $5 \pm 1 \mathrm{~nm}$ are green-gray.

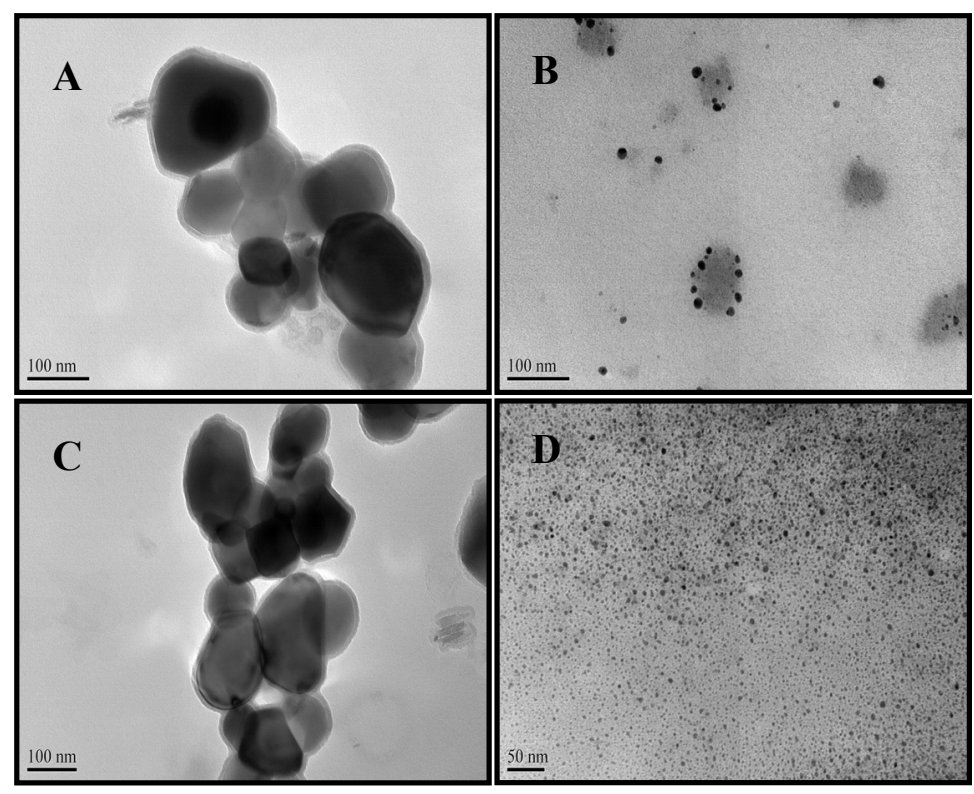

The effect of the irradiation time on the enhancements obtained in the SERS experiments using the metallic NPs prepared was evaluated using 4-ABT for Ag NPs. Similarly, 1,2-bis(4-pyridyl)ethylene (BPE) was used to evaluate the optimum conditions for the synthesis of Au NPs. These analytes were selected based on the affinity of the compounds with the corresponding NPs. Figure 3 displays the SERS spectrum results of Au NPs at different irradiation times. The best enhancement in the Raman signals for 4-ABT was observed at 20 min of irradiation time for Ag and Au NPs. The optimized parameter (20 min of irradiation) was used during all subsequent syntheses in this work. Aliquots of $5 \mu \mathrm{L}$ of NP suspensions at different times of analysis were deposited on Au slides of approximately $0.25 \mathrm{~cm}^{2}$. The drops of NP suspensions were allowed to dry in a desiccator overnight, and then, the analyte was deposited on the substrate and again placed in a desiccator.

Predominant vibrational signals were observed in the Raman spectrum of 4-ABT at 1140, 1390 and $1430 \mathrm{~cm}^{-1}$. These signals can be attributed to modes assigned to the $9 \mathrm{~b}, 3$ and $19 \mathrm{~b}$ modes of the b2-type ring, respectively. The low intensity band at $1080 \mathrm{~cm}^{-1}$ is due to the 7 a mode of the a1-type ring [33-37]. The main Raman bands of BPE are observed in SERS spectrum of the probe molecules deposited on Au NPs. The peak at $994 \mathrm{~cm}^{-1}$ corresponds to the ring breathing mode of BPE pyridine. A blue shift of $27 \mathrm{~cm}^{-1}$ was observed in the SERS spectra of BPE, which includes the vibrational movement of the pyridyl nitrogen atom. Similarly, the vibrational signature observed at $1596 \mathrm{~cm}^{-1}$ corresponds to the $\mathrm{C}-\mathrm{N}$ stretching mode of the pyridyl ring. This band presents a blue shift of approximately $10 \mathrm{~cm}^{-1}$ in the SERS spectrum of BPE deposited on Au NPs. These results suggest that the molecule of BPE interacts strongly with the surface of the Au NPs through the nitrogen atom corresponding to the pyridyl ring [38]. However, the bands at $1637 \mathrm{~cm}^{-1}$ and $1200 \mathrm{~cm}^{-1}$ remain unshifted. 
The size distribution of the gold and silver nanoparticles was evaluated. The analysis was conducted to verify how the irradiation time during the laser ablation synthesis affected the average size of the NPs. The results are shown in Table 1. The average particle size distributions of Au and Ag NPs synthesized by different ablation times are shown. The particles at 5 min are larger $(96 \mathrm{~nm})$ than those obtained at $20 \mathrm{~min}$. As the ablation time increases from 5 to $20 \mathrm{~min}$, the size distribution experiences a significant decrease of NP size and the average particle size is reduced to $75 \mathrm{~nm}$. Similar results were obtained by Baladi [39] in the synthesis of Al nanoparticles [40,41].

Figure 3. Surface enhanced Raman scattering (SERS) spectrum of (A) $1 \mathrm{mM} \mathrm{BPE}$ in Au NPs at various irradiation times. Raman and SERS spectra were acquired at $785 \mathrm{~nm}$. (B) $1 \mathrm{mM}$ 4-ABT deposited on Ag NPs deposited on Au-coated glass slide at various irradiation times. SERS spectra were acquired at $532 \mathrm{~nm}$.

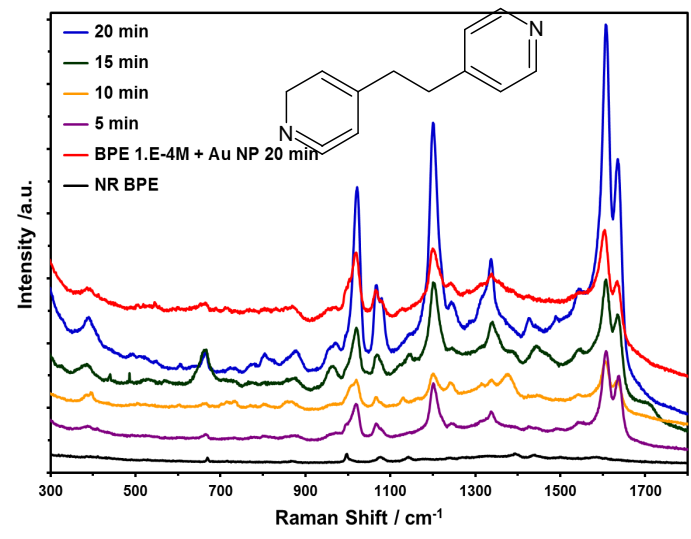

(A) BPE

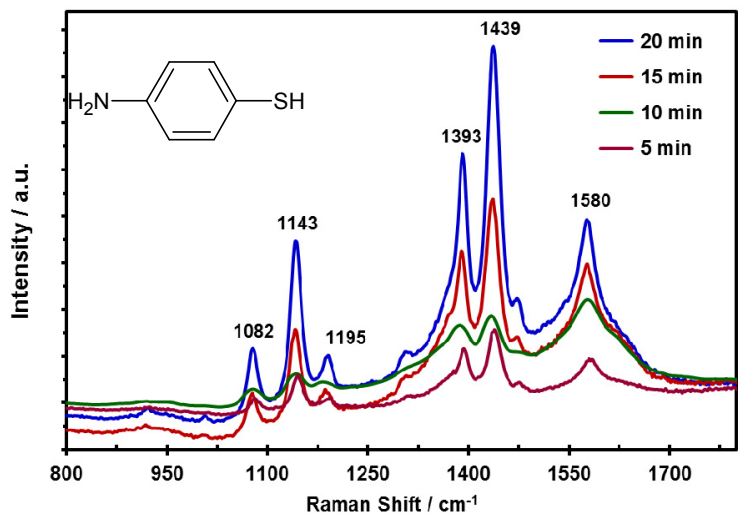

(B) 4-ABT

Table 1. Average size of Au and Ag NPs synthesized by laser ablation.

\begin{tabular}{ccc}
\hline Nanoparticle Type & Irradiation Time (min) & Average Size (Z size, nm) \\
\hline $\mathrm{Au}$ & 5 & 96 \\
$\mathrm{Au}$ & 10 & 82 \\
$\mathrm{Au}$ & 15 & 71 \\
$\mathrm{Au}$ & 20 & 75 \\
$\mathrm{Ag}$ & 5 & 77 \\
$\mathrm{Ag}$ & 20 & 66 \\
\hline
\end{tabular}

The $\mathrm{pH}$ plays a very important role in the properties of the NPs [42] prepared, including their SERS activity [43]. A change in the ionic strength in the medium leads to the formation of clusters of particles or even a monolayer of particles on a surface. This conglomeration of NPs leads to changes in their color analogous to the variation in color associated with altering the size or the shape of the particles. When two or more particles stick together, the absorption produced is very similar to that of a single rod-like particle with a larger length [44]. Water at different $\mathrm{pH}$ values was used to evaluate the SERS activity of the NPs. The $\mathrm{pH}$ of the water was adjusted to 2.6, 4.8, 8.1 and 10.3. The irradiation time used for the syntheses was $20 \mathrm{~min}$. The colors of the NPs in water depend on the $\mathrm{pH}$ value. Figure 4 contains color micrographs of Au and Ag NP suspensions synthesized at the various 
$\mathrm{pH}$ values studied. Differences in the predominant colloidal color were found. These color changes can be associated with the average size and the predominant shapes of the NPs in the suspensions.

Figure 4. NPs suspensions at different $\mathrm{pH}$ values of the solvent during synthesis: (A) $\mathrm{Au}$ NPs suspensions; (B) Ag NPs suspensions.

(A)

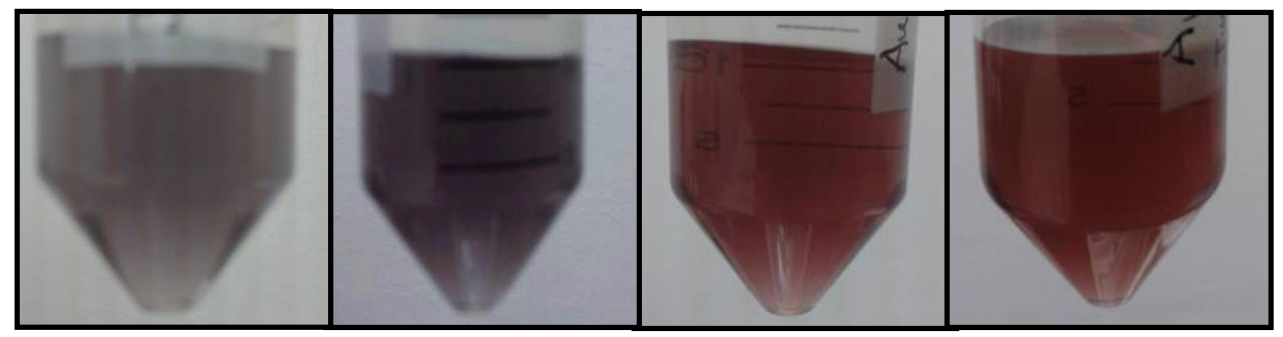

$\mathrm{pH}: 2.6 \quad 4.88 .110 .3$

(B)

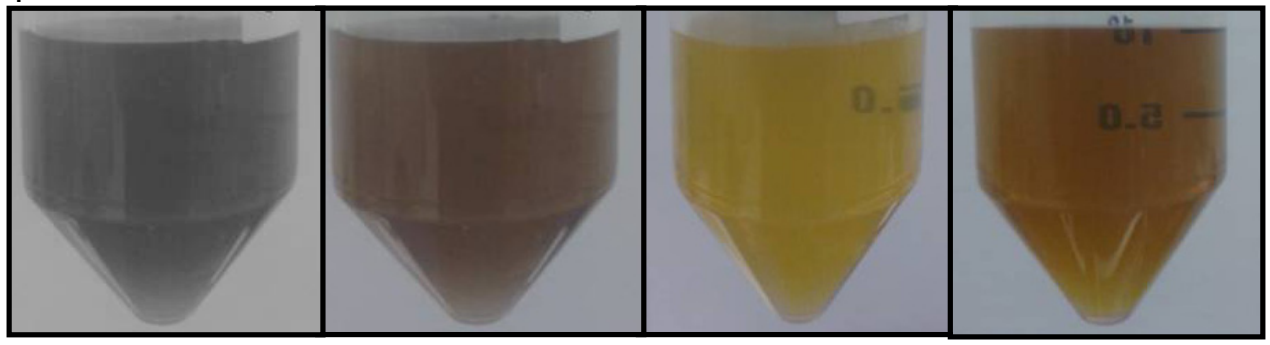

Figure 5 shows the UV-Vis absorption spectra for Au and Ag nanoparticles synthesized at different $\mathrm{pH}$ values. A broad absorption band indicates the presence of different sizes coexisting in the colloidal suspension. At acidic pH values, a low absorbance was found for both Au and Ag NP suspensions. Therefore, at $\mathrm{pH}$ values of 8.1 (slightly basic) and 10.3 (basic), intense absorbances were exhibited by the suspensions. No changes in the characteristic wavelength location of the plasmon resonance absorption bands were detected for the metallic NPs under study.

$\mathrm{Au}$ or Ag NPs suspensions at different $\mathrm{pH}$ values were transferred onto gold-coated glass slides to prepare the SERS substrates. Then, the samples were allowed to dry, and $5.0 \mu \mathrm{L}$ of $1 \mathrm{mM} 4-\mathrm{ABT}$ was deposited on the substrates. The SERS measurements were acquired using a Raman excitation source at $532 \mathrm{~nm}$ for the analytes deposited on Ag NPs and $785 \mathrm{~nm}$ for the Au NPs substrates. The results for 4-ABT on $\mathrm{Au}$ and Ag NPs are shown in Figure 6.

Figure 5. UV-Vis absorption spectra of NPs colloids after synthesis at various $\mathrm{pH}$ : (A) $\mathrm{Au} ;(\mathbf{B}) \mathrm{Ag}$.

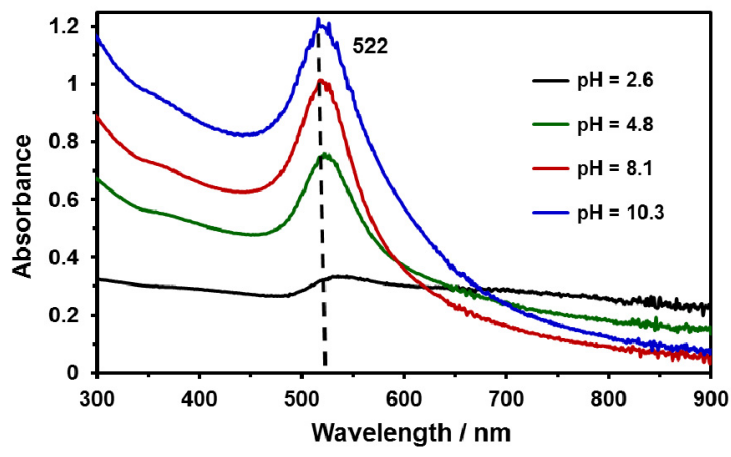

(A) $\mathrm{Au}$

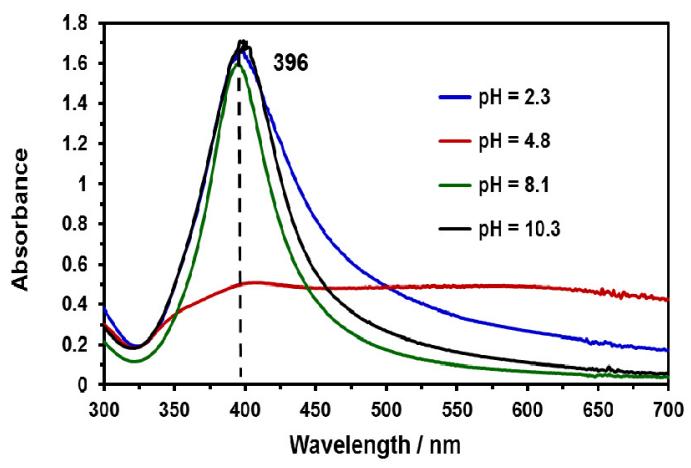

(B) Ag 
Figure 6. $\mathrm{pH}$ effect on SERS activity: (A) SERS spectra of 4-ABT on the Au NPs/Au substrate; (B) SERS spectra of 4-ABT on the Ag NPs/Au substrate; (C) TNT SERS spectra on the Au NPs/Au substrate; and (D) SERS spectra of TNT interacting with a Au colloidal suspension, included for comparison.

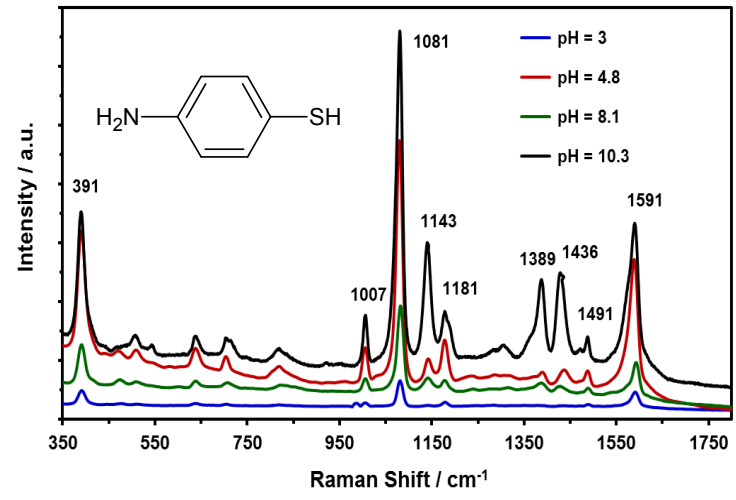

(A) 4-ABT/AuNP

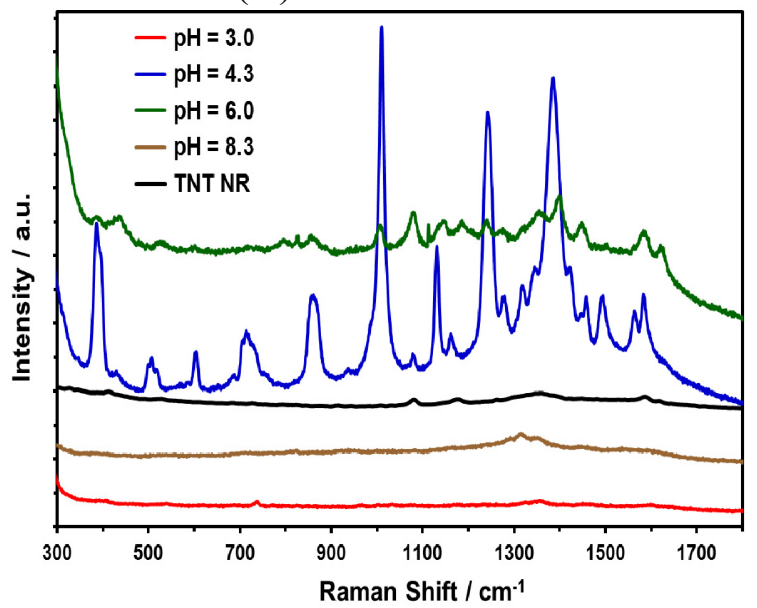

(C) TNT/AuNP

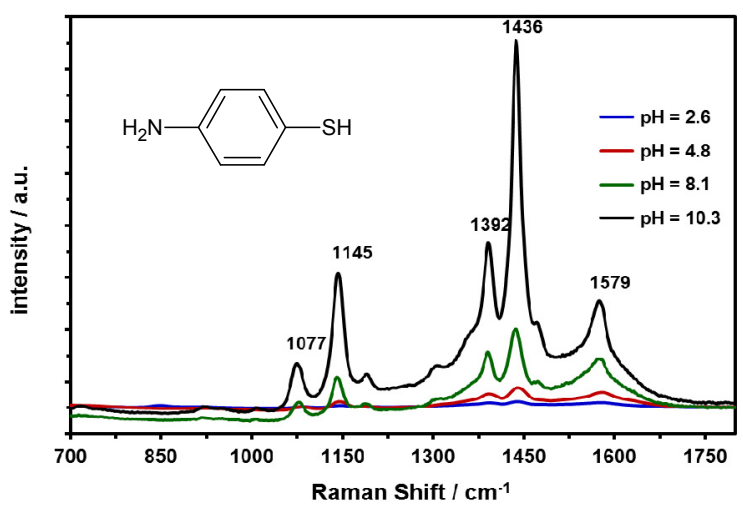

(B) 4-ABT/AgNP

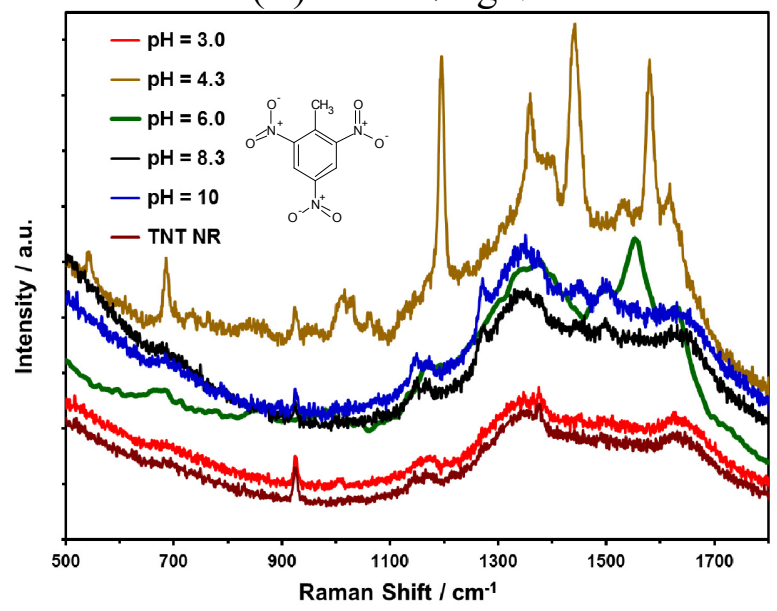

(D) TNT/Au-COLL

The SERS spectra of 4-ABT on Au NPs shown in Figure 6A are in good agreement with previous results [32]. The four strong peaks at $1591,1436,1389$ and $1143 \mathrm{~cm}^{-1}$ can be assigned to ring $8 \mathrm{~b}, 19 \mathrm{~b}$, 3 and $9 \mathrm{~b}$ modes of 4-ABT, respectively. The peaks at Raman shifts of 1491 and $1081 \mathrm{~cm}^{-1}$ are due to $8 \mathrm{a}$ and 19a modes, respectively, that possess the a1-type of symmetry [26,45]. For Au NPs, the best results were found at $\mathrm{pH}$ values of 10.3 and 4.8. Kim and collaborators found that the b2-type bands of 4-ABT are strongly affected by the solution $\mathrm{pH}$ [33]. Regardless of the excitation wavelength and the type of SERS substrates, the b2-type bands appeared very weak or negligible at acidic $\mathrm{pH}$, while they were observed very distinctly at basic $\mathrm{pH}$ in Ag NPs. Our results show differences in the signals at 1143 and $1181 \mathrm{~cm}^{-1}$ for Au NPs at different $\mathrm{pH}$ values. Kim [46] attributes the disappearance of the b2-type bands at acidic $\mathrm{pH}$ to the protonation of the amine group, thus causing the charge transfer resonance chemical enhancement to be less likely to occur.

Likewise, the results for 4-ABT obtained for Ag NPs deposited on Au-coated glass slides presented good enhancement in the Raman signals, specifically the NP suspensions prepared at basic $\mathrm{pH}$. Figure 6B shows the Raman signals for $1 \mathrm{mM} 4-\mathrm{ABT}$. To determine the surface enhancement factor (SEF) for 4-ABT, aliquots of $1.0 \times 10^{-9} \mathrm{M}$ were deposited on the NP substrates. The deposited sample covered an area of approximately of $0.25 \mathrm{~cm}^{2}$. If the spot of the laser using an objective of $10 \times$ is 
approximately $13 \mu \mathrm{m}^{2}$, the number of 4-ABT molecules that were illuminated was calculated to be $1.4 \times 10^{5}$, which represents a SEF of $1.6 \times 10^{9}$.

To determine the effect of $\mathrm{pH}$ on Au NPs in SERS activity for the detection of TNT, $3 \mu \mathrm{L}$ of a solution of the explosive at $1.17 \mathrm{mM}$ was deposited on different Au substrates with gold NPs deposited. The NPs were synthesized at various $\mathrm{pH}$ values. The best results were observed at a $\mathrm{pH}$ value of nanoparticles of 4.3 and 6.0. The particles obtained at acidic $\mathrm{pH}(\sim 3)$ were very unstable for $\mathrm{Au}$ NPs. The NPs were precipitated approximately $1 \mathrm{~h}$ after preparation. Colloidal results are shown in Figure 6D to compare the results of nanoparticles deposited versus colloidal suspensions of nanoparticles. A poor enhancement is observed in the colloidal suspension at $\mathrm{pH}$ 4.3. The results confirm one of the intrinsic limitations of metal colloidal nanoparticles for SERS applications, where their robustness as SERS substrates is compromised. An important factor in the enhancement obtained through SERS is the need for aggregation to generate the necessary plasmonic conditions for the production of significant SERS. If adequate aggregation does not occur, the reproducibility of SERS on colloidal NPs is affected because the kinetics of the process can be uncontrollable once aggregation begins.

SERS is an important technique to develop applications for the detection of highly energetic materials (HEM) [47-54]. TNT is a HEM of vast military applications and uses. TNT was selected as a nitroaromatic HEM to evaluate Au NPs on different substrates. Solutions containing TNT were transferred onto various substrates containing Au NPs that were deposited and immobilized. Substrates, such as Al and quartz plates, were also used to deposit Au NPs. TNT solutions at different concentrations $\left(1.0 \times 10^{-4} \mathrm{M}, 1.0 \times 10^{-6} \mathrm{M}\right.$ and $\left.1.0 \times 10^{-10} \mathrm{M}\right)$ were used to determine the lowest amount of HEM that could be quantitatively detected (Low Limit of Detection, LOD). The results shown in Figure 7 indicate an increase in the intensity of the principal vibrational signals of TNT due to SERS, which presents strong bands.

Figure 7. SERS spectra of TNT deposited on Au NPs; spectra were taken at $785 \mathrm{~nm}$ : (A) NPs were deposited on different substrates (Al film, Au film and quartz); (B) TNT deposited at different concentrations and $\mathrm{Al}$ was used as the substrate.

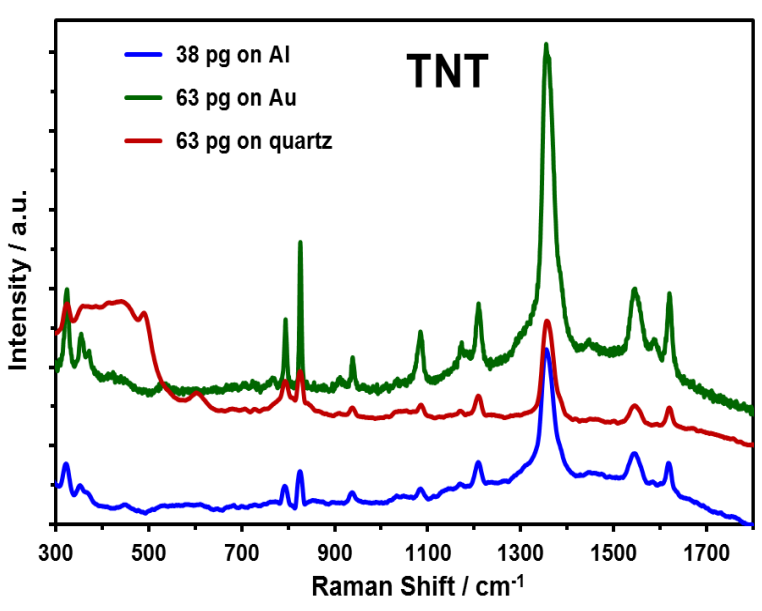

(A)

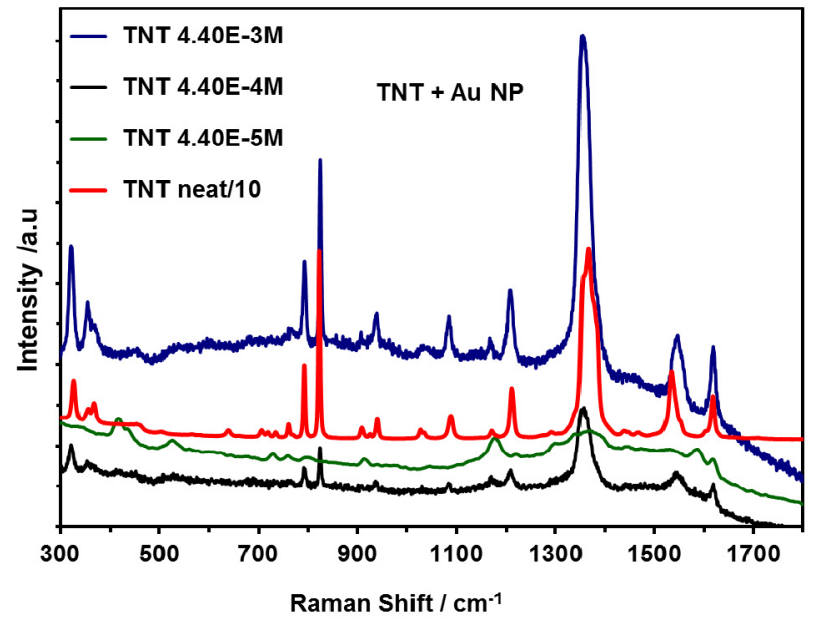

(B) 
The signal observed corresponded to the presence of the $\mathrm{NO}_{2}$ group out-of-plane bending mode at $826 \mathrm{~cm}^{-1}$ and the $\mathrm{NO}_{2}$ stretching mode at $1300-1370 \mathrm{~cm}^{-1}$ [55]. Primera-Pedrozo et al. reported similar results in 2008 [31], where the enhancement was obtained from colloidal gold NPs with a modification of the ionic strength of the media that was used to interact with the explosive. A SEF of $2 \times 10^{9}$ was obtained for TNT.

The zeta potential of a system is a measure of the charge stability and controls all particle-particle interactions within a suspension. Understanding the zeta potential is of critical importance in controlling the dispersion and determining the stability of a nanoparticle suspension, i.e., to what degree aggregation will occur over time [56]. A lower level of the zeta potential results $(0$ to $\pm 30 \mathrm{mV})$ in a smaller electrostatic repulsion between the particles, maximizing aggregation/flocculation. Zeta potential measurements of the as-prepared samples yielded values of $-34.9 \mathrm{mV}$ for the Au colloid and $-20.9 \mathrm{mV}$ for the Ag colloid, thus confirming the moderate stability of gold and silver nanoparticles. Similarly, the Z-potential and the Z-size were acquired for colloidal suspensions of Ag and Au NPs at different $\mathrm{pH}$ values. The results confirm the color differences in the colloidal suspension when the $\mathrm{pH}$ was adjusted to the solutions before the synthesis. The results are shown in Table 2. The values of the $\mathrm{Z}$ potential reflect that the stability of the Au NPs is compromised at different $\mathrm{pH}$ values of the synthesis of colloidal suspensions. For this reason, the suspended colloidal NPs result in low reproducibility, lower SEF values and higher LODs.

Table 2. Results of the Z-potential and the Z-size for Au NPs and Ag NPs at various $\mathrm{pH}$ values.

\begin{tabular}{cccc}
\hline NP Type & pH & Average Size (nm) & Z Potential (mV) \\
\hline $\mathrm{Ag}$ & 4.2 & 96 & -18.0 \\
$\mathrm{Ag}$ & 6.0 & 90 & -21.8 \\
$\mathrm{Ag}$ & 8.3 & 87 & -24.1 \\
$\mathrm{Ag}$ & 10.0 & 72 & -26.2 \\
$\mathrm{Ag}$ & 10.8 & 70 & -29.1 \\
$\mathrm{Au}$ & 4.3 & 93 & -11.1 \\
$\mathrm{Au}$ & 6.0 & 75 & -39.2 \\
$\mathrm{Au}$ & 8.3 & 73 & -3.7 \\
$\mathrm{Au}$ & 10.0 & 47 & -5.2 \\
\hline
\end{tabular}

Moreover, we have determined the number of TNT molecules that were present on the Au NPs for an area of approximately of $1.3 \times 10^{7} \mu \mathrm{m}^{2}$ deposited on a substrate. The interrogation area (circular) or laser spot when using a $20 \times$ objective was $2.3 \times 10^{3} \mu \mathrm{m}^{2}$. The TNT mass contained in the interrogated area for the highest concentration solution was $7.8 \times 10^{-12} \mathrm{~g}$. The number of TNT molecules that were SERS excited was calculated to be $2.1 \times 10^{10}$ molecules $\left(1.0 \times 10^{-4} \mathrm{M}, 2 \mu \mathrm{L}\right.$ deposited $)$, $2.1 \times 10^{8}$ molecules $\left(1.0 \times 10^{-6} \mathrm{M}, 2 \mu \mathrm{L}\right)$ and $2.1 \times 10^{4}$ molecules $\left(1.0 \times 10^{-10} \mathrm{M}, 2 \mu \mathrm{L}\right)$.

The data were compared with experiments using colloidal NPs. The Raman signals were not observed in colloidal NPs. A possible explanation is that the acquisition of good enhanced Raman signals depends on the reliability and stability of the SERS-active sites (or "hot spots"), which have a large influence on the enhancement of the Raman signal intensities. However, the enhancement in the signals obtained depends significantly on the aggregation of Ag or Au colloids and the analyte used in 
the analysis. The stability of metal colloids is due to the repulsive forces derived from the charged species on the surface of the colloidal particles, which assume a nonzero effective charge. When these charges are replaced with a neutral adsorbate, aggregation occurs, usually when a ligand has a greater affinity for the metal than that of the surface charged species [57].

\section{Conclusions}

Colloidal suspensions of $\mathrm{Ag}$ and $\mathrm{Au}$ NPs were successfully synthesized by laser ablation using water as the solvent. Colloids of different colors and sizes were obtained, depending on the time of irradiation in the synthesis and the $\mathrm{pH}$ of water. Excellent SERS results were found for Au and Ag NPs deposited on Au films using 4-ABT at a $\mathrm{pH}$ value of 10.3.

The potential application of $\mathrm{Au}$ and $\mathrm{Ag}$ nanoparticles in SERS detection of explosives was evaluated. We have detected $7.8 \times 10^{-18} \mathrm{~g}$ of TNT on Au NPs deposited on Al sheets. The SEF obtained establishes the possibility of using the substrates prepared for the detection of contaminants in water, such as nitroaromatic HEM.

\section{Acknowledgments}

Support from the U.S. Department of Homeland Security under Award Number 2008-ST-061-ED0001 is acknowledged. However, the views and conclusions contained in this document are those of the authors and should not be considered a representation of the official policies, either expressed or implied, of the U.S. Department of Homeland Security.

This contribution was supported by the U.S. Department of Defense, Proposal No: 58949-PH-REP, Agreement No: W911NF-11-1-0152. The authors also acknowledge contributions from Richard T. Hammond from the Army Research Office, DOD.

\section{References}

1. Hamouda, I.M. Current perspectives of nanoparticles in medical and dental biomaterials. J. Biomed. Res. 2012, 26, 143-151.

2. Albrecht, M.; Janke, V.; Sievers, S.; Siegner, U.; Schüler, D.; Heyen, U. Scanning force microscopy study of biogenic nanoparticles for medical applications. J. Magn. Magn. Mater. 2005, 290-291, 269-271.

3. Rai, M.; Yadav, A.; Gade, A. Silver nanoparticles as a new generation of antimicrobials. Biotechnol. Adv. 2009, 27, 76-83.

4. Piao, M.J.; Kang, K.A.; Lee, I.K.; Kim, H.S.; Kim, S.; Choi, J.Y.; Choi, J.; Hyun, J.W. Silver nanoparticles induce oxidative cell damage in human liver cells through inhibition of reduced glutathione and induction of mitochondria-involved apoptosis. Toxicol. Lett. 2011, 201, 92-100.

5. Chen, X.; Schluesener, H.J. Nanosilver: A nanoproduct in medical application. Toxicol. Lett. 2008, 176, 1-12.

6. Wei, D.; Ye, Y.; Jia, X.; Yuan, C.; Qian, W. Chitosan as an active support for assembly of metal nanoparticles and application of the resultant bioconjugates in catalysis. Carbohydr. Res. 2010, $345,74-81$. 
7. Lee, K.Y.; Hwang, J.; Lee, Y.W.; Kim, J.; Han, S.W. One-step synthesis of gold nanoparticles using azacryptand and their applications in SERS and catalysis. J. Colloid Interface Sci. 2007, 316, 476-481.

8. Hutchings, G.J.; Edwards, J.K. Application of Gold Nanoparticles in Catalysis. Chapter 6. In Frontiers of Nanoscience; Roy, L.J., Wilcoxon, J.P., Eds.; Elsevier: Amsterdam, Netherlands, 2012; Volume 3, pp. 249-293.

9. Niu, Y.; Crooks, R.M. Dendrimer-encapsulated metal nanoparticles and their applications to catalysis. Comptes Rendus Chimie 2003, 6, 1049-1059.

10. Chang, C.-C.; Yang, K.-H.; Liu, Y.-C.; Hsu, T.-C. New pathway to prepare gold nanoparticles and their applications in catalysis and surface-enhanced Raman scattering. Colloids Surf. B 2012, 93, 169-173.

11. Yang, J.; Pan, J. Hydrothermal synthesis of silver nanoparticles by sodium alginate and their applications in surface-enhanced Raman scattering and catalysis. Acta Mater. 2012, 60, 4753-4758.

12. Eriksson, S.; Nylén, U.; Rojas, S.; Boutonnet, M. Preparation of catalysts from microemulsions and their applications in heterogeneous catalysis. Appl. Catal. A 2004, 265, 207-219.

13. Haruta, M. Catalysis and applications of gold nanoparticles. In Studies in Surface Science and Catalysis; Masakazu Anpo, M.O., Hiromi, Y., Eds.; Elsevier: Amsterdam, Netherlands, 2003; Volume 145, pp. 31-38.

14. Chen, X.; Zhu, H. 3.01-Catalysis by Supported Gold Nanoparticles. In Comprehensive Nanoscience and Technology; David, L.A., Gregory, D.S., Gary, P.W., Eds.; Academic Press, Elsevier: Amsterdam, Netherlands, 2011; pp. 1-11.

15. El-Rafie, M.H.; Shaheen, T.I.; Mohamed, A.A.; Hebeish, A. Bio-synthesis and applications of silver nanoparticles onto cotton fabrics. Carbohydr. Polym. 2012, 90, 915-920.

16. Jung, S.-H.; Kim, K.-I.; Ryu, J.-H.; Choi, S.-H.; Kim, J.-B.; Moon, J.-H.; Jin, J.-H. Preparation of radioactive core-shell type 198Au@ $\mathrm{SiO}_{2}$ nanoparticles as a radiotracer for industrial process applications. Appl. Radiat. Isot. 2010, 68, 1025-1029.

17. Yu, C.H.; Oduro, W.; Tam, K.; Tsang, E.S.C. Chapter 10. Some Applications of Nanoparticles. In Handbook of Metal Physics; John, A.B., Ed.; Elsevier: Amsterdam, Netherlands, 2008; Volume 5, pp. 365-380.

18. Ganesh, S.; Amit, S. Perspectives on the Science and Technology of Nanoparticle Synthesis. In Nanomaterials Handbook; CRC Press: Boca Raton, FL, USA, 2006.

19. Lawrence, D.Ä.; Ryan, R. Synthesis of Metal Colloids. In Catalyst Preparation; CRC Press: New York, NY, USA, 2006; pp. 93-137.

20. Sergeev, G.B. 2-Synthesis and Stabilization of Nanoparticles. In Nanochemistry; Elsevier Science: Amsterdam, Netherlands, 2006; pp. 7-36.

21. Takeshi, T. Preparation of Nanoparticles Using Laser Ablation in Liquids. In Laser Ablation in Liquids; Pan Stanford Publishing: Singapore, 2012; pp. 207-268.

22. Haibo, Z.; Shikuan, Y.; Weiping, C. Formation of Nanoparticles under Laser Ablation of Solids in Liquids. In Laser Ablation in Liquids; Pan Stanford Publishing: Singapore, 2012; pp. 327-396.

23. Stratakisa, E.; Shafeevb, G. Nanostructures? Formation under Laser Ablation of Solids in Liquids. In Laser Ablation in Liquids, Pan Stanford Publishing: Singapore, 2012; pp. 815-854. 
24. López-Tocón, I.; Centeno, S.P.; Otero, J.C.; Marcos, J.I. Selection rules for the charge transfer enhancement mechanism in SERS: Dependence of the intensities on the L-matrix. J. Mol. Struct. 2001, 565-566, 369-372.

25. Luo, Z.; Fang, Y. Investigation of the mechanism of influence of colloidal gold/silver substrates in nonaqueous liquids on the surface enhanced Raman spectroscopy (SERS) of fullerenes C60 (C70). J. Colloid Interface Sci. 2006, 301, 184-192.

26. Primera-Pedrozo, O.M.; Rodríguez, G.D.M.; Castellanos, J.; Felix-Rivera, H.; Resto, O.; Hernández-Rivera, S.P. Increasing surface enhanced Raman spectroscopy effect of RNA and DNA components by changing the $\mathrm{pH}$ of silver colloidal suspensions. Spectrochim. Acta A $\mathbf{2 0 1 2}$, $87,77-85$.

27. Hou, X.; Zhang, X.; Chen, S.; Fang, Y.; Li, N.; Zhai, X.; Liu, Y. Size-controlled synthesis of Au nanoparticles and nanowires and their application as SERS substrates. Colloids Surf. A 2011, 384, 345-351.

28. Kundu, S.; Mandal, M.; Ghosh, S.K.; Pal, T. Photochemical deposition of SERS active silver nanoparticles on silica gel and their application as catalysts for the reduction of aromatic nitro compounds. J. Colloid Interface Sci. 2004, 272, 134-144.

29. Photopoulos, P.; Boukos, N.; Panagopoulou, M.; Meintanis, N.; Pantiskos, N.; Raptis, Y.; Tsoukalas, D. Size control of Ag nanoparticles for SERS sensing applications. Procedia. Eng. 2011, 25, 280-283.

30. Vlckova, B.; Pavel, I.; Sladkova, M.; Siskova, K.; Slouf, M. Single molecule SERS: Perspectives of analytical applications. J. Mol. Struct. 2007, 834-836, $42-47$.

31. Primera-Pedrozo, O.M.; Jerez-Rozo, J.I.; De La Cruz-Montoya, E.; Luna-Pineda, T.; Pacheco-Londoño, L.C.; Hernandez-Rivera, S.P. Nanotechnology-based detection of explosives and biological agents simulants. Sens. J. 2008, 8, 963-973.

32. Burda, C.; Chen, X.; Narayanan, R.; El-Sayed, M.A. Chemistry and properties of nanocrystals of different shapes. Chem. Rev. 2005, 105, 1025-1102.

33. Kim, K.; Shin, D.; Choi, J.-Y.; Kim, K.L.; Shin, K.S. Surface-enhanced Raman scattering characteristics of 4 -aminobenzenethiol derivatives adsorbed on silver. J. Phys. Chem. C 2011, $115,24960-24966$.

34. Kim, K.; Lee, H.B.; Choi, J.-Y.; Kim, K.L.; Shin, K.S. Surface-enhanced Raman scattering of 4-aminobenzenethiol in nanogaps between a planar Ag substrate and Pt nanoparticles. J. Phys. Chemistry C 2011, 115, 13223-13231.

35. Kim, K.; Kim, K.L.; Shin, D.; Choi, J.-Y.; Shin, K.S. Surface-enhanced Raman scattering of 4-aminobenzenethiol on $\mathrm{Ag}$ and $\mathrm{Au}: \mathrm{pH}$ dependence of b2-type bands. J. Phys. Chem. C 2012, 116, 4774-4779.

36. Kim, K.; Kim, K.L.; Lee, H.B.; Shin, K.S. Similarity and dissimilarity in surface-enhanced Raman scattering of 4-aminobenzenethiol, 4,4'-dimercaptoazobenzene, and 4,4'-dimercaptohydrazobenzene on Ag. J. Phys. Chem. C 2012, 116, 11635-11642.

37. Kim, K.; Yoon, J.K.; Lee, H.B.; Shin, D.; Shin, K.S. Surface-enhanced Raman scattering of 4-aminobenzenethiol in Ag sol: Relative intensity of a1- and b2-type bands invariant against aggregation of Ag nanoparticles. Langmuir 2011, 27, 4526-4531. 
38. Kim, A.; Ou, F.S.; Ohlberg, D.A.A.; Hu, M.; Williams, R.S.; Li, Z. Study of molecular trapping inside gold nanofinger arrays on surface-enhanced Raman substrates. J. American Chem. Soc. 2011, 133, 8234-8239.

39. Baladi, A.; Sarraf Mamoory, R. Investigation of different liquid media and ablation times on pulsed laser ablation synthesis of aluminum nanoparticles. Appl. Surf. Sci. 2010, 256, 7559-7564.

40. Mahfouz, R.; Cadete Santos Aires, F.J.; Brenier, A.; Jacquier, B.; Bertolini, J.C. Synthesis and physico-chemical characteristics of nanosized particles produced by laser ablation of a nickel target in water. Appl. Surf. Sci. 2008, 254, 5181-5190.

41. Tsuji, T.; Iryo, K.; Watanabe, N.; Tsuji, M. Preparation of silver nanoparticles by laser ablation in solution: Influence of laser wavelength on particle size. Appl. Surf. Sci. 2002, 202, 80-85.

42. Riabinina, D.; Zhang, J.; Chaker, M.; Margot, J.; Ma, D. Size control of gold nanoparticles synthesized by laser ablation in liquid media. ISRN Nanotechnol. 2012, 2012, 5.

43. Cañamares, M.V.; Garcia-Ramos, J.V.; Gómez-Varga, J.D.; Domingo, C.; Sanchez-Cortes, S. Comparative study of the morphology, aggregation, adherence to glass, and surface-enhanced Raman scattering activity of silver nanoparticles prepared by chemical reduction of $\mathrm{Ag}^{+}$using citrate and hydroxylamine. Langmuir 2005, 21, 8546-8553.

44. Schatz, S.H.G. Synthesis and Analysis of SILVER/GOLD Nanoparticles. Available online: http://nanohub.org/topics/GeneralChemistry/File:Silvergold_module.pdf (accessed on 10 December 2012)

45. Kim, K.; Lee, H.S. Effect of Ag and Au nanoparticles on the SERS of 4-aminobenzenethiol assembled on powdered copper. J. Phys. Chem. B 2005, 109, 18929-18934.

46. Kim, K. Surface-Enhanced Raman Scattering Characteristics of 4-Aminobenzenethiol Derivatives Adsorbed on Silver. Available online: http://ipc.iisc.ernet.in/ umalab/icors2012/Mo_109.pdf (accessed on 10 December 2012)

47. Liu, X.; Zhao, L.; Shen, H.; Xu, H.; Lu, L. Ordered gold nanoparticle arrays as surface-enhanced Raman spectroscopy substrates for label-free detection of nitroexplosives. Talanta 2011, 83, 1023-1029.

48. Kawaguchi, T.; Shankaran, D.R.; Kim, S.J.; Matsumoto, K.; Toko, K.; Miura, N. Surface plasmon resonance immunosensor using Au nanoparticle for detection of TNT. Sens. Actuators B 2008, 133, 467-472.

49. Kneipp, K.; Wang, Y.; Dasari, R.R.; Feld, M.S.; Gilbert, B.D.; Janni, J.; Steinfeld, J.I. Near-infrared surface-enhanced Raman scattering of trinitrotoluene on colloidal gold and silver. Spectrochim. Acta A 1995, 51, 2171-2175.

50. Lin, D.; Liu, H.; Qian, K.; Zhou, X.; Yang, L.; Liu, J. Ultrasensitive optical detection of trinitrotoluene by ethylenediamine-capped gold nanoparticles. Anal. Chim. Acta 2012, 744, 92-98.

51. Izake, E.L. Forensic and homeland security applications of modern portable Raman spectroscopy. Forensic Sci. Int. 2010, 202, 1-8.

52. Caygill, J.S.; Davis, F.; Higson, S.P.J. Current trends in explosive detection techniques. Talanta 2012, 88, 14-29.

53. Fierro-Mercado, P.; Renteria-Beleño, B.; Hernández-Rivera, S.P. Preparation of SERS-active substrates using thermal inkjet technology. Chem. Phys. Lett. 2012, 552, 108-113. 
54. Fierro-Mercado, P.; Hernández-Rivera, S.P. Highly sensitive filter paper substrate for SERS trace explosives detection. Int. J. Spectrosc. 2012, 2012, doi:10.1155/2012/716527.

55. Jerez Rozo, J.I.; Chamoun, A.M.; Peña, S.L.; Hernández-Rivera, S.P. Enhanced Raman scattering of TNT on nanoparticle substrates: Ag colloids prepared by reduction with hydroxylamine hydrochloride and sodium citrate. Proc. SPIE 2007, 6538, 653824-653835.

56. Cosgrove, T. Colloid Science Principles, Methods and Applications; Blackwell Publishing Ltd: Department of Chemistry, University of Bristol, Bristol, UK, 2005.

57. Muniz-Miranda, M.; Pergolese, B.; Bigotto, A.; Giusti, A. Stable and efficient silver substrates for SERS spectroscopy. J. Colloid Interface Sci. 2007, 314, 540-544.

(C) 2013 by the authors; licensee MDPI, Basel, Switzerland. This article is an open access article distributed under the terms and conditions of the Creative Commons Attribution license (http://creativecommons.org/licenses/by/3.0/). 